\title{
Study of the analgesic activities, chronic toxicity and addictive potential of Jia-Yuan-Qing pill in rats
}

\author{
YE TIAN $^{1}$, LI-RONG TENG ${ }^{1}$, ZHEN-ZUO WANG $^{1}$, MIN ZHAO $^{1}$, QING-FAN MENG ${ }^{1}$, JIA-HUI LU ${ }^{1}$, \\ JIAN-MING TIAN ${ }^{2}$, WEI-WEI ZHANG ${ }^{2}$, XIAOYI ZHENG ${ }^{3}$, DI WANG ${ }^{1}$ and LE-SHENG TENG ${ }^{1}$ \\ ${ }^{1}$ College of Life Sciences, Jilin University, Changchun, Jilin 130012; ${ }^{2}$ Jilin Academy of Traditional Chinese Medicine, \\ Changchun, Jilin 130012, P.R. China; ${ }^{3}$ Department of Nephrology, School of Medicine, \\ Stanford University, Stanford, CA 94305, USA
}

Received April 25, 2014; Accepted December 12, 2014

DOI: $10.3892 /$ etm.2015.2426

\begin{abstract}
Jia-Yuan-Qing pill (JYQP) composed of Porcellio laevis Latreille, Corydalis Rhizoma and Radix Cynanchi Paniculati at a ratio of 9:7:7 has been found to be an effective analgesic agent. The present study aimed to evaluate the safety, addictive potential and anti-cancer pain activity of JYQP in a rat model. During the 6-month chronic toxicity test, no significant changes in general behavior, defecation, postural abnormalities, dietary or water intake or blood biochemical parameters were observed in male and female rats. Although a high dose of JYQP $(5 \mathrm{~g} / \mathrm{kg})$ caused swelling of the liver, spleen and kidney in male and female rats, no pathological changes were observed in all organs examined via hematoxylin and eosin staining. The analgesic effect of JYQP on bone cancer pain was successfully confirmed in a rat model of Walker 256 cell-induced bone cancer. In contrast to morphine, in a physical dependence test, JYQP produced no withdrawal symptoms following chronic administration. The data from this study provide experimental evidence supporting the clinical use of JYQP as an effective, safe and non-addictive agent for the treatment of bone cancer pain.
\end{abstract}

\section{Introduction}

It has been reported that approximately one-third of patients who are actively receiving treatment for cancer, and two-thirds of those with advanced malignant disease experience pain (1). Cancer pain, particularly bone cancer pain, which is one of the most frequent symptoms in bone cancer patients (2), affects

Correspondence to: Dr Le-Sheng Teng or Dr Di Wang, College of Life Sciences, Jilin University, 2699 Qianjin Avenue, Changchun, Jilin 130012, P.R. China

E-mail: tenglesheng@jlu.edu.cn

E-mail: jluwangdi@gmail.com

Key words: traditional Chinese medicine, Jia-Yuan-Qing pill, bone cancer pain, long-term toxicity, psychological addiction their quality of life, and current treatments are limited (3). Carcinomas and sarcomas of various types metastasize to bone, resulting in spontaneous bone pain, hyperalgesia (exaggerated pain) and allodynia (pain in response to a normally innocuous stimulus), in addition to the degradation of bone and remodeling of peripheral nerves (4). Although opiates, particularly the opioid receptor agonist morphine, have been used as conventionally prescribed analgesic drugs for the treatment of advanced bone cancer pain, patients often undergo tolerance to analgesics as well as dose-limiting side-effects, which limit their long-term clinical application (5). Thus, new drugs that are effective and have minimal side-effects are urgently required.

The efficacy of traditional Chinese medicine (TCM) is recognized in numerous countries $(6,7)$. Confirmed by clinical trials and experimental studies, certain TCMs possess remarkable efficacies in pain treatment, including Tou-Feng-Yu pill (8) and Tong-Luo-San-Jie gel (9). Moreover, surveys have revealed that TCMs are used as important auxiliary therapeutics among $41-62 \%$ of cancer patients $(9,10)$. Following traditional Chinese prescriptions, Porcellio laevis Latreille, Corydalis Rhizoma and Radix Cynanchi Paniculati have been combined at a ratio of 9:7:7 to provide a novel formulation named Jia-Yuan-Qing pill (JYQP). A patent for the JYQP formula has been authorized in China (Patent No. 201110147689.7) (11). As reported previously, Porcellio laevis Latreille (12), Corydalis Rhizoma (13) and Radix Cynanchi Paniculati (14) have been used as analgesic drugs for many years. Our study performed in mice has demonstrated that JYQP exerts its analgesic activity via the participation of peripheral nerve (15).

Although TCMs are generally accepted to be safe and efficacious for long-term use, pharmacological and toxicological evaluations for TCMs are necessary (16). There have been no previous studies demonstrating the safety of the three herbal components (Porcelain laevis Latreille, Corydalis Rhizoma and Radix Cynanchi Paniculati) of JYQP and no chronic toxicity tests on rats have been reported.

The aim of the present study was to evaluate the safety, addictive potential and analgesic effect on cancer pain of JYQP in rats. After 6 months of JYQP administration, bodyweight changes, blood biochemical parameters and organ coefficients were monitored. The activity of JYQP on cancer 
pain was assessed in a rat model of bone cancer pain induced by the Walker 256 cell line. The withdrawal behavior was also assessed in comparison with morphine.

\section{Materials and methods}

Materials. The Porcellio laevis Latreille, Corydalis Rhizoma and Radix Cynanchi Paniculati components of JYQP were supplied by the College of Pharmaceutical Science, Jilin University (Changchun, China). As recommended in the Chinese Pharmacopoeia (17), JYQP was prepared as follows: Porcellio laevis Latreille (90 g) and Radix Cynanchi Paniculati $(70 \mathrm{~g})$ powders were immersed and boiled in a 10 -fold volume of distilled water for $1.5 \mathrm{~h} .70 \mathrm{~g}$ Corydalis Rhizoma powder was boiled in $0.4170 \%(\mathrm{v} / \mathrm{v})$ ethanol for $1.0 \mathrm{~h}$. These processes were repeated twice. All the extracts were filtered, mixed, concentrated and finally spray-dried. The dry powder (yield of $21.1 \%, \mathrm{w} / \mathrm{w})$ was used for bioactivity determination.

Animals. Wistar rats were obtained from the Experimental Animal Center of Jilin University, and were housed in a regulated environment $\left(20 \pm 2^{\circ} \mathrm{C}\right)$, with a $12 \mathrm{~h}$ light/dark cycle (08:00 a.m. to 08:00 p.m., light). The animals were deprived of food for $12 \mathrm{~h}$ prior to the experiment, with free access to drinking water. Each animal was used only once in the experiment. All animal treatments were strictly in accordance with international ethical guidelines concerning the care and use of laboratory animals, and all the experiments were carried out under the approval of the Committee of Experimental Animal Administration of Jilin University [License NO.: SCXK-(JI) 2011-0004]. Each experimental group consisted of 10 animals.

\section{Long-term toxicity study}

Healthy male and female rats (70-90 g) were randomly divided into four pairs of groups (10 males and 10 females, respectively, in each pair). Rats were orally administered JYQP $(0$, $0.5,2.5$ or $5.0 \mathrm{~g} / \mathrm{kg}$ per day) for six months. All animals were monitored daily for mortality and clinical signs including general condition, skin and fur, bodyweight, dietary and water intake, defecation and behavioral patterns.

Blood biochemical analysis. At the last day of the experiment, rat blood samples were collected and 13 biochemical parameters listed as follows were detected using an automatic biochemical analyzer (Cobas 8000; Roche Diagnostics $\mathrm{GmbH}$, Mannheim, Germany): alanine transaminase, aspartate transaminase, total protein, albumin, total bilirubin, $\gamma$-glutamyltranspeptidase, alkaline phosphatase, blood urea nitrogen, creatinine, creatine kinase, glucose, triglycerides and total cholesterol. Plasma was used for GLU determination, while serum was used for analysis of other parameters.

Histopathological assessment. The rats were sacrificed at the end of the experiment by the administration of $200 \mathrm{mg} / \mathrm{kg}$ pentobarbital. The organs including brain, heart, lungs, liver, spleen, kidneys, adrenal glands, thymus, prostate gland, epididymis, testis, uterus and ovary were collected, weighed and fixed in $10 \%$ buffered formalin solution. After paraffin-embedding and sectioning, hematoxylin and eosin staining was performed to analyze the pathological alterations of the organs. Paired organs were weighed together.
Bone cancer pain test

Bone cancer pain model establishment. Walker 256 carcinoma cells $\left[1 \times 10^{7}\right.$ cells $/ \mathrm{ml}$; American Type Culture Collection (ATCC), Manassas, VA, USA; passage <10] were intraperitoneally injected into 80 male and female rats (150-170 g). After 6-7 days, the cells in the ascitic fluid were collected, and resuspended at a concentration of $3.5 \times 10^{5}$ cells $/ 6 \mu 1$ in phosphate-buffered saline (PBS). The bone cancer pain model was established according to the method previously described (9). In brief, following anesthetization with pentobarbital $(50 \mathrm{mg} / \mathrm{kg}$ ), under aseptic conditions, right lateral superficial incisions were made in the skin covering the patella of rats. A 23 -gauge needle was inserted into the tibia medullary cavity through the intercondylar eminence $(7 \mathrm{~mm}$ below the knee joint). Either Walker 256 carcinoma cells $\left(3.5 \times 10^{5}\right)$ in $6 \mu 1$ PBS or $6 \mu \mathrm{l}$ PBS alone was injected through the hole using a 10- $\mu 1$ micro injection syringe. The injection site was closed using bone wax. After recovery, the animals were returned to their cages.

Rat foot-supporting force test. This test was carried out using a rat foot-holding power instrument (YLS-11A; Yi Yan Technology Development Co., Ltd., Jinan, China). A total of 50 bone cancer model rats were randomly divided into five groups ( $n=10 /$ group). They were orally treated with vehicle $(10 \mathrm{ml} / \mathrm{kg})$, JYQP $(0.5,1.2$ or $2.4 \mathrm{~g} / \mathrm{kg})$ or Tramadol $(20 \mathrm{mg} / \mathrm{kg}$ ). Then, $60 \mathrm{~min}$ later, the rats were transferred onto the footboards of the supporting force instrument. The angle between the rat body and the footboard surface was kept constant. The tail of the rat was placed between the two support plates, while the head and the body of the rat were kept in alignment. The anchorage forces of the left and right legs were read respectively and the difference between them was calculated.

Mechanical threshold. The same drug administration protocol as mentioned above was performed. At $60 \mathrm{~min}$, the sensitivity to mechanical stimuli of each rat was examined. The right foot paw of the rat was placed on the electronic instrument (YLS-3E; Yi Yan Technology Development Co., Ltd.) to measure tenderness, and then another paw was tested. The pyramidal indenter was allowed to slowly fall down, and the indenter vertex was aimed at the center of the paw. When the rat began to struggle, the pyramidal indenter was stopped and data were recorded.

Physical dependence. Rats were assessed for physical dependence on morphine and JYQP. The experimental process is shown in (Fig. 1). Healthy rats (180-200 g) were randomly divided into five pairs of groups (10 males and 10 females, respectively, in each pair). The rats were treated with morphine and JYQP twice a day for 30 days. Bodyweight was measured weekly. On day 31, the administration of morphine and JYQP was terminated to assess physical dependence. The rats were placed in separate transparent cages $(25 \mathrm{~cm}$ diameter x $30 \mathrm{~cm}$ height) and the number of precipitated withdrawal scratches, wet-dog shakes, fur caring, and physical characteristics were measured within $24 \mathrm{~h}$.

Statistical analysis. Statistical analysis was performed using SPSS 16.0 software (SPSS, Inc., Chicago, IL, USA). One-way variance analysis (ANOVA) was used to determine statistical 


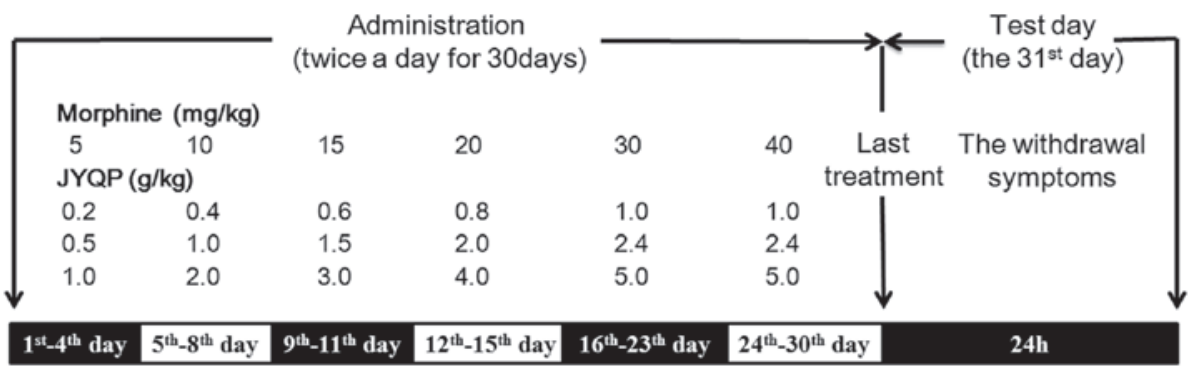

Figure 1. Experimental protocol for the assessment of physical dependence on Jia-Yuan-Qing pill (JYQP). Details are described in Materials and methods.

A

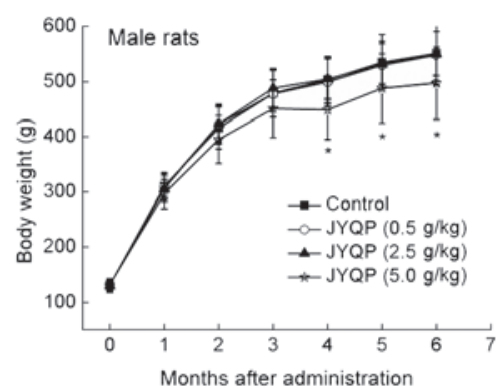

C

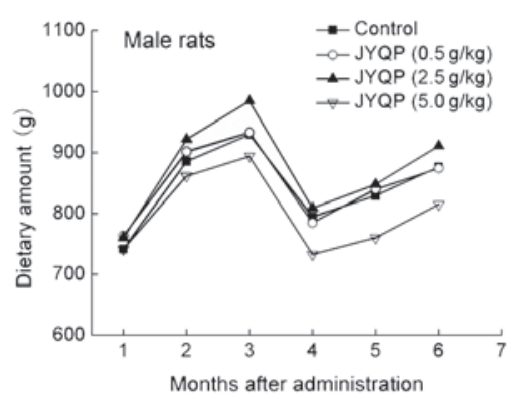

$\mathbf{E}$

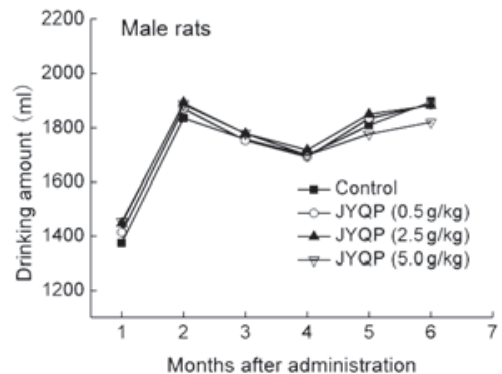

B

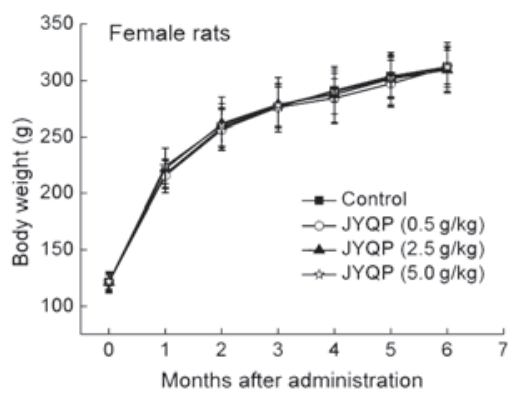

D

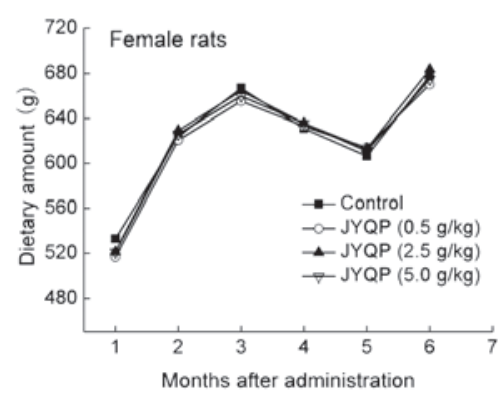

$\mathbf{F}$

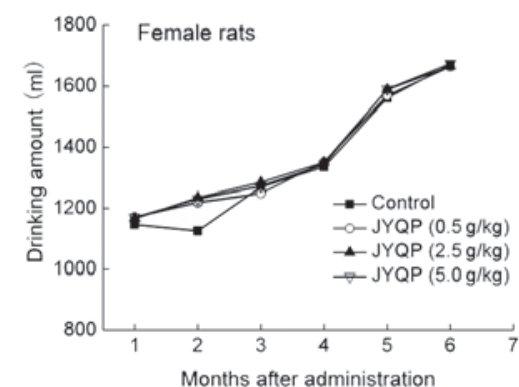

Figure 2. Changes of (A and B) body weight, (C and D) dietary amount and (E and F) drinking amount for male and female rats, respectively, treated with Jia-Yuan-Qing pill (JYQP; 0.5, 2.5 or $5.0 \mathrm{~g} / \mathrm{kg}$ ) for 6 months. Data are expressed as mean \pm SD (n=10/group) and analyzed using one-way analysis of variance. ${ }^{*} \mathrm{P}<0.05$ versus control rats.

significance, followed by post-hoc multiple comparisons (Dunn's test). Data are expressed as mean \pm standard deviation (SD). Statistical significance was defined as $\mathrm{P}<0.05$.

\section{Results}

Long-term toxicity examination. No deaths were observed among the rats during the 6-month administration of JYQP at the doses of $0.5,2.5$ and $5.0 \mathrm{~g} / \mathrm{kg}$. However, salivation was noted in male and female rats treated with 2.5 and $5.0 \mathrm{~g} / \mathrm{kg} \mathrm{JYQP}$ from week three. No further changes in general behaviors or physiological activities were observed in the JYQP-treated rats.
Compared with the control rats, from week 4 , a loss of bodyweight in $5.0 \mathrm{~g} / \mathrm{kg}$ JYQP-treated male rats was noted $(\mathrm{P}<0.05 ;$ Fig. 2A). No significant differences among the groups in dietary and water intakes were observed (Fig. 2).

As shown in Table I, the organ coefficients in the control and JYQP-treated rats were analyzed. The weights of the liver, spleen and kidneys were increased in male and female rats after 6 months of JYQP administration at a dose of $5.0 \mathrm{~g} / \mathrm{kg}$ compared with those in control rats $(\mathrm{P}<0.05$; Table I). However, hematoxylin and eosin staining revealed that no pathologic changes had occurred in the organs (data not shown). Furthermore, in comparison with the control group, the blood 
Table I. Organ coefficient results after 6-month Jia-Yuan-Qing pill (JYQP) administration in rats.

\begin{tabular}{|c|c|c|c|c|}
\hline \multirow[b]{2}{*}{ Organs } & \multirow[b]{2}{*}{ Control } & \multicolumn{3}{|c|}{ JYQP (g/kg) } \\
\hline & & 0.5 & 2.5 & 5.0 \\
\hline \multicolumn{5}{|l|}{ Male } \\
\hline Heart (g) & $0.32 \pm 0.049$ & $0.30 \pm 0.055$ & $0.30 \pm 0.022$ & $0.30 \pm 0.022$ \\
\hline Liver (g) & $2.49 \pm 0.552$ & $2.41 \pm 0.371$ & $2.47 \pm 0.242$ & $3.03 \pm 0.493^{\mathrm{a}}$ \\
\hline Spleen (g) & $0.17 \pm 0.038$ & $0.18 \pm 0.052$ & $0.19 \pm 0.086$ & $0.21 \pm 0.051^{\mathrm{a}}$ \\
\hline Lung (g) & $0.41 \pm 0.046$ & $0.41 \pm 0.049$ & $0.39 \pm 0.044$ & $0.40 \pm 0.070$ \\
\hline Kidneys (g) & $0.56 \pm 0.098$ & $0.61 \pm 0.158$ & $0.61 \pm 0.050$ & $0.66 \pm 0.103^{a}$ \\
\hline Adrenals (mg) & $14.73 \pm 1.905$ & $13.96 \pm 4.443$ & $14.59 \pm 3.215$ & $16.63 \pm 2.585$ \\
\hline Thymus (g) & $0.07 \pm 0.019$ & $0.08 \pm 0.029$ & $0.08 \pm 0.015$ & $0.07 \pm 0.018$ \\
\hline Prostate (g) & $0.17 \pm 0.047$ & $0.18 \pm 0.030$ & $0.18 \pm 0.030$ & $0.17 \pm 0.041$ \\
\hline Testis (g) & $0.68 \pm 0.088$ & $0.70 \pm 0.138$ & $0.69 \pm 0.074$ & $0.69 \pm 0.071$ \\
\hline Epididymis (g) & $0.38 \pm 0.081$ & $0.42 \pm 0.090$ & $0.38 \pm 0.075$ & $0.42 \pm 0.059$ \\
\hline Brain $(\mathrm{g})$ & $0.39 \pm 0.025$ & $0.37 \pm 0.059$ & $0.37 \pm 0.042$ & $0.40 \pm 0.065$ \\
\hline \multicolumn{5}{|l|}{ Female } \\
\hline Heart (g) & $0.32 \pm 0.023$ & $0.34 \pm 0.033$ & $0.32 \pm 0.045$ & $0.32 \pm 0.028$ \\
\hline Liver (g) & $2.26 \pm 0.219$ & $2.39 \pm 0.169$ & $2.37 \pm 0.325$ & $2.49 \pm 0.252^{\mathrm{a}}$ \\
\hline Spleen (g) & $0.16 \pm 0.021$ & $0.18 \pm 0.019$ & $0.18 \pm 0.031$ & $0.21 \pm 0.026^{\mathrm{b}}$ \\
\hline Lung (g) & $0.46 \pm 0.059$ & $0.46 \pm 0.084$ & $0.49 \pm 0.081$ & $0.47 \pm 0.063$ \\
\hline Kidneys (g) & $0.55 \pm 0.062$ & $0.58 \pm 0.034$ & $0.56 \pm 0.051$ & $0.67 \pm 0.064^{b}$ \\
\hline Adrenals (mg) & $36.11 \pm 3.868$ & $34.86 \pm 5.832$ & $35.12 \pm 4.561$ & $35.53 \pm 7.702$ \\
\hline Thymus (g) & $0.10 \pm 0.019$ & $0.11 \pm 0.016$ & $0.11 \pm 0.034$ & $0.11 \pm 0.023$ \\
\hline Ovary (g) & $0.069 \pm 0.021$ & $0.064 \pm 0.014$ & $0.070 \pm 0.023$ & $0.069 \pm 0.018$ \\
\hline Uterus (g) & $0.236 \pm 0.063$ & $0.267 \pm 0.064$ & $0.270 \pm 0.070$ & $0.264 \pm 0.082$ \\
\hline Brain (g) & $0.56 \pm 0.060$ & $0.57 \pm 0.120$ & $0.57 \pm 0.053$ & $0.56 \pm 0.039$ \\
\hline
\end{tabular}

Data are presented as mean $\pm \mathrm{SD}\left(\mathrm{n}=10\right.$ per group); ${ }^{\mathrm{a}} \mathrm{P}<0.05,{ }^{\mathrm{b}} \mathrm{P}<0.001$ vs. the control group.

biochemical parameters in all JYQP treated rats exhibited no significant changes ( $\mathrm{P}>0.05$; Table II).

Bone cancer pain test. In the foot-supporting force test, the differences of bipedal pressure were notably higher in the rats from the bone cancer model group than in the vehicle-treated rats. Tramadol $(20 \mathrm{mg} / \mathrm{kg})$ and JYQP $(0.5,1.2$ and $2.4 \mathrm{~g} / \mathrm{kg})$ produced a significant antinociceptive effect on bone cancer pain, indicated by the reduction of the bipedal pressure difference ( $\mathrm{P}<0.05$; Fig. 3A).

In the analysis of mechanical allodynia, the vehicle-treated rats with bone cancer pain displayed a profound reduction of mechanical threshold compared with that of the sham group ( $\mathrm{P}<0.01$; Fig. 3B). Treatment with Tramadol $(20 \mathrm{mg} / \mathrm{kg})$ or JYQP $(0.5,1.2,2.4 \mathrm{~g} / \mathrm{kg})$ strongly increased the mechanical threshold in the model rats compared with that in the vehicle-treated group (Fig. 3B).

Physical dependence. When the JYQP-treated rats were compared with non-treated rats, JYQP exhibited no significant influence on bodyweight; however, 30 days of morphine administration resulted $>9 \%$ bodyweight loss in male ( $\mathrm{P}<0.05$; Fig. 4A) and female rats $(\mathrm{P}<0.05$; Fig. 4B).
Withdrawal behaviors such as stretching, wet-dog shakes and fur carding were quantified to assess the physical dependence on morphine and JYQP. Morphine-dependent rats displayed extremely high withdrawal behaviors; however, in the JYQP-treated rats, no withdrawal symptoms were noted, indicating that JYQP is a reliable and safe agent without addictive properties (Fig. 5).

\section{Discussion}

The present study aimed to investigate the safety, addictive potential and analgesic effect on cancer pain of JYQP. Toxicity assessment is the most important data for new drug registration, and the safety of herbal products is of concern to the medical community and public (18). In the present study, a chronic toxicity test in Wistar rats was performed to examine the safety of JYQP. The rats were treated with JYQP at dose of $0,0.5,2.5$ or $5.0 \mathrm{~g} / \mathrm{kg}$ for 6 months, and no significant changes on general behavior, defecation, postural abnormalities, dietary or water intake, and blood biochemical parameters were observed in male and female rats. Changes in bodyweight have served as an indicator for drug toxicity (19). Although $5 \mathrm{~g} / \mathrm{kg}$ JYQP caused bodyweight loss after 4 weeks 
Table II. Effect on blood biochemical parameters of Jia-Yuan-Qing pill (JYQP) administration in rats.

\begin{tabular}{|c|c|c|c|c|}
\hline \multirow[b]{2}{*}{ Biochemical parameters } & \multirow[b]{2}{*}{ Control } & \multicolumn{3}{|c|}{ JYQP (g/kg) } \\
\hline & & 0.5 & 2.5 & 5.0 \\
\hline \multicolumn{5}{|l|}{ Male } \\
\hline $\operatorname{ALT}(\mathrm{U} / \mathrm{l})$ & $45.64 \pm 11.26$ & $45.83 \pm 11.02$ & $49.77 \pm 12.27$ & $45.80 \pm 14.40$ \\
\hline AST (U/l) & $114.83 \pm 9.11$ & $114.59 \pm 16.31$ & $117.15 \pm 11.05$ & $110.53 \pm 19.05$ \\
\hline $\mathrm{TP}(\mathrm{g} / \mathrm{l})$ & $63.15 \pm 3.82$ & $60.76 \pm 2.86$ & $61.21 \pm 2.24$ & $61.84 \pm 2.43$ \\
\hline $\operatorname{ALB}(\mathrm{g} / \mathrm{l})$ & $34.63 \pm 2.54$ & $33.49 \pm 1.50$ & $33.04 \pm 1.97$ & $33.39 \pm 1.52$ \\
\hline T-BIL $(\mu \mathrm{mol} / 1)$ & $4.49 \pm 0.93$ & $4.20 \pm 1.09$ & $4.34 \pm 0.78$ & $4.29 \pm 0.80$ \\
\hline GGT (U/1) & $2.91 \pm 0.45$ & $3.18 \pm 0.77$ & $3.20 \pm 1.01$ & $3.08 \pm 0.73$ \\
\hline ALP (U/l) & $63.63 \pm 11.44$ & $58.91 \pm 9.88$ & $60.66 \pm 10.94$ & $66.95 \pm 12.35$ \\
\hline BUN (mmol/1) & $6.74 \pm 0.70$ & $6.74 \pm 0.69$ & $6.19 \pm 0.60$ & $6.49 \pm 0.57$ \\
\hline CRE $(\mu \mathrm{mol} / 1)$ & $83.09 \pm 9.27$ & $82.11 \pm 6.39$ & $78.63 \pm 4.32$ & $80.02 \pm 7.03$ \\
\hline $\mathrm{CK}(\mathrm{U} / \mathrm{l})$ & $555.71 \pm 367.22$ & $664.32 \pm 309.97$ & $615.24 \pm 272.85$ & $530.79 \pm 238.06$ \\
\hline GLU (mmol/l) & $5.20 \pm 1.25$ & $5.43 \pm 0.73$ & $5.12 \pm 0.64$ & $5.08 \pm 1.00$ \\
\hline $\mathrm{TG}(\mathrm{mmol} / \mathrm{l})$ & $0.63 \pm 0.24$ & $0.60 \pm 0.19$ & $0.64 \pm 0.21$ & $0.75 \pm 0.42$ \\
\hline $\mathrm{TC}(\mathrm{mmol} / \mathrm{l})$ & $1.60 \pm 0.34$ & $1.41 \pm 0.30$ & $1.61 \pm 0.28$ & $1.43 \pm 0.29$ \\
\hline \multicolumn{5}{|l|}{ Female } \\
\hline $\operatorname{ALT}(\mathrm{U} / \mathrm{l})$ & $33.52 \pm 6.33$ & $36.17 \pm 6.84$ & $34.08 \pm 6.50$ & $38.16 \pm 6.36$ \\
\hline AST (U/l) & $102.06 \pm 18.17$ & $105.66 \pm 18.27$ & $100.43 \pm 10.09$ & $112.75 \pm 20.97$ \\
\hline $\mathrm{TP}(\mathrm{g} / \mathrm{l})$ & $63.19 \pm 3.25$ & $65.00 \pm 3.89$ & $64.65 \pm 2.15$ & $64.54 \pm 2.96$ \\
\hline $\operatorname{ALB}(g / l)$ & $37.51 \pm 2.09$ & $38.01 \pm 2.41$ & $38.15 \pm 2.53$ & $36.00 \pm 2.41$ \\
\hline T-BIL $(\mu \mathrm{mol} / \mathrm{l})$ & $4.18 \pm 0.99$ & $4.10 \pm 0.88$ & $4.38 \pm 0.60$ & $4.35 \pm 0.53$ \\
\hline GGT (U/l) & $2.66 \pm 0.30$ & $2.86 \pm 0.45$ & $2.71 \pm 0.54$ & $2.84 \pm 0.75$ \\
\hline $\operatorname{ALP}(\mathrm{U} / \mathrm{l})$ & $30.36 \pm 9.05$ & $29.09 \pm 5.88$ & $34.82 \pm 5.52$ & $36.67 \pm 7.26$ \\
\hline BUN (mmol/l) & $6.98 \pm 0.70$ & $6.64 \pm 1.19$ & $6.56 \pm 0.43$ & $6.98 \pm 0.98$ \\
\hline $\mathrm{CRE}(\mu \mathrm{mol} / \mathrm{l})$ & $78.99 \pm 6.64$ & $80.78 \pm 6.03$ & $79.85 \pm 4.67$ & $78.86 \pm 6.09$ \\
\hline $\mathrm{CK}(\mathrm{U} / \mathrm{l})$ & $454.39 \pm 142.23$ & $450.98 \pm 176.98$ & $422.40 \pm 111.25$ & $491.37 \pm 223.43$ \\
\hline GLU (mmol /l) & $5.61 \pm 0.64$ & $5.75 \pm 0.74$ & $5.86 \pm 1.06$ & $5.27 \pm 1.44$ \\
\hline TG (mmol /l) & $0.33 \pm 0.11$ & $0.27 \pm 0.05$ & $0.36 \pm 0.10$ & $0.40 \pm 0.11$ \\
\hline $\mathrm{TC}(\mathrm{mmol} / \mathrm{l})$ & $1.49 \pm 0.43$ & $1.51 \pm 0.38$ & $1.31 \pm 0.33$ & $1.39 \pm 0.28$ \\
\hline
\end{tabular}

Data are presented as mean $\pm \mathrm{SD}$ ( $\mathrm{n}=10$ per group). ALT, alanine transaminase; AST, aspartate transaminase; TP, total protein; ALB, albumin; T-BIL, total bilirubin; GGT, $\gamma$-glutamyltranspeptidase; ALP, alkaline phosphatase; BUN, blood urea nitrogen; CRE, creatinine; CK, creatine kinase; GLU, glucose; TG, triglycerides; TC, total cholesterol.

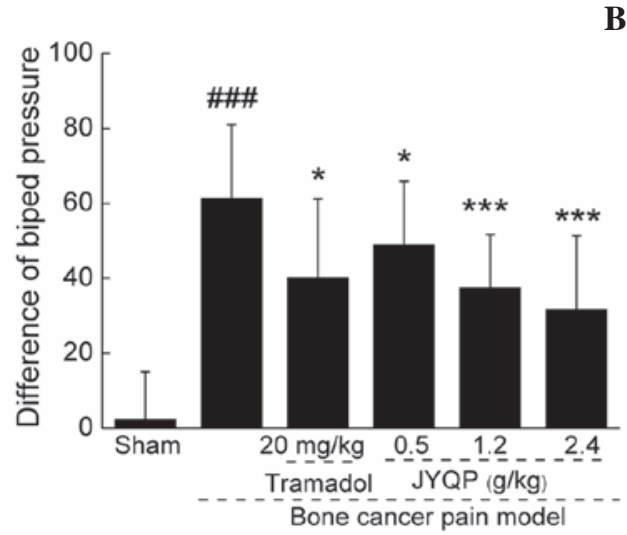

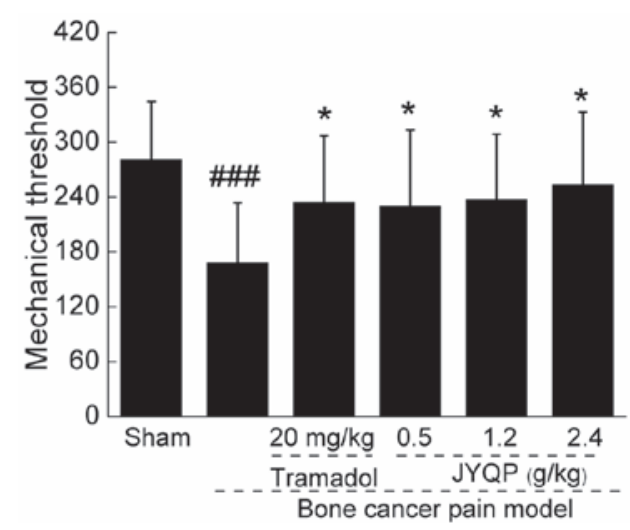

Figure 3. Analgesic activities of Jia-Yuan-Qing pill (JYQP) in (A) the foot-supporting force and (B) mechanical threshold tests in a rat bone cancer model. Rats with bone cancer were orally treated with saline $(10 \mathrm{ml} / \mathrm{kg})$, JYQP $(0.5,1.2$ and $2.4 \mathrm{~g} / \mathrm{kg})$ or tramadol $(20 \mathrm{mg} / \mathrm{kg}) .60 \mathrm{~min}$ later, the two tests were performed, respectively. Data are expressed as mean $\pm \mathrm{SD}\left(\mathrm{n}=10 /\right.$ group) and were analyzed using one-way analysis of variance $(\mathrm{ANOVA}){ }^{*} \mathrm{P}<0.05$ and ${ }^{* * *} \mathrm{P}<0.001 \mathrm{vs}$. model rats; ${ }^{\# \#} \mathrm{P}<0.001$ vs. sham rats. 
A

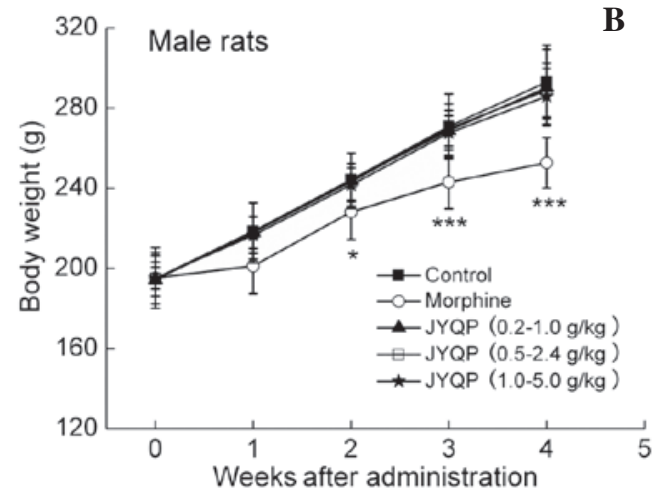

B

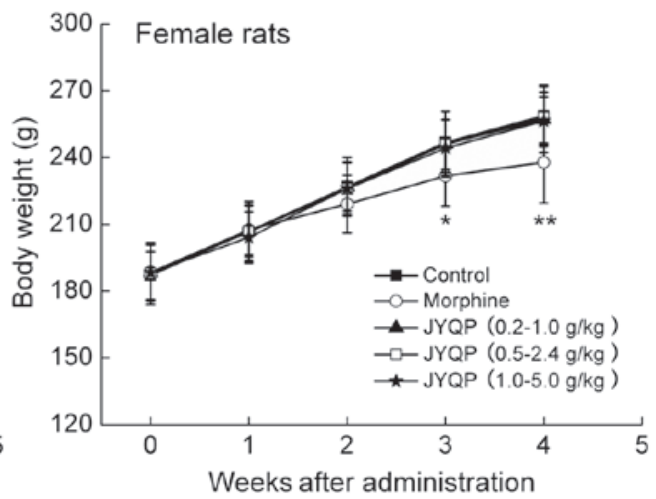

Figure 4. Bodyweight changes of (A) male and (B) female rats treated with morphine or Jia-Yuan-Qing pill (JYQP) were measured individually. Data are expressed as mean $\pm \mathrm{SD}$ ( $\mathrm{n}=10 /$ group of male or female rats) and analyzed using one-way analysis of variance (ANOVA). ${ }^{*} \mathrm{P}<0.05,{ }^{* * *} \mathrm{P}<0.01,{ }^{* * *} \mathrm{P}<0.001$ vs. non-treated rats.

A

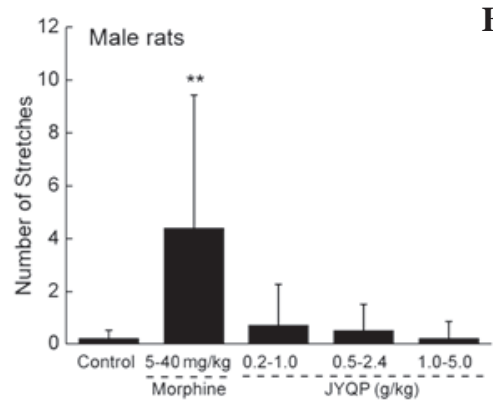

C

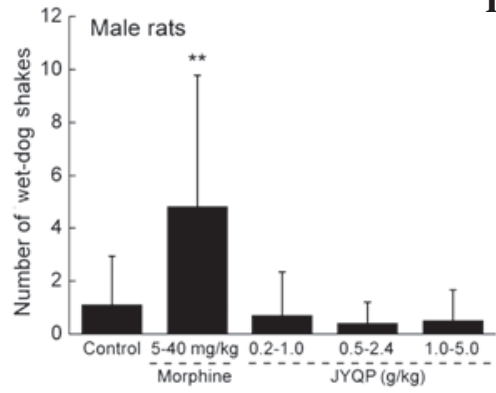

E

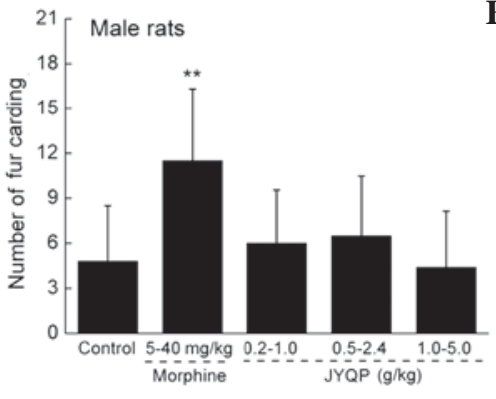

B

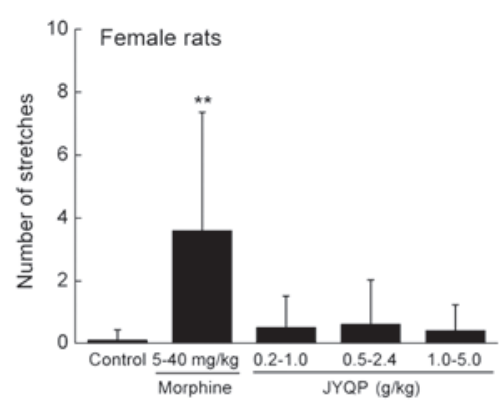

D

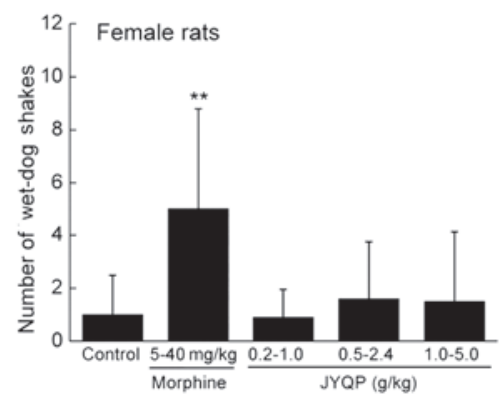

F

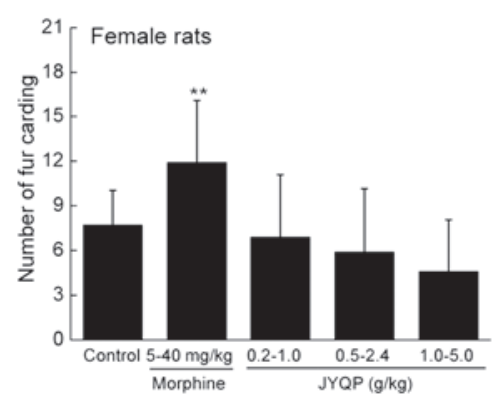

Figure 5. Withdrawal symptoms of rats in the physical dependence test. Behaviors including (A and B) stretching, (C and D) wet-dog shakes and (E and F) fur carding in JYQP or morphine-treated male and female rats were monitored for $24 \mathrm{~h}$ after drug withdrawal. Data are expressed as mean $\pm \mathrm{SD}$ ( $\mathrm{n}=10 / \mathrm{group})$ and analyzed using one-way ANOVA. ${ }^{* *} \mathrm{P}<0.01$ vs. non-treated rats.

of administration to male rats, this dose was nearly 10 -fold higher than the effective dose. Moreover, following the administration of $5 \mathrm{~g} / \mathrm{kg}$ JYQP for 6 months, swelling of the liver, spleen and kidney were observed in male and female rats; however, no pathological changes were observed in any of the organs via hematoxylin and eosin staining (data not shown). The weight changes of the liver, spleen and kidney caused by the high dose of JYQP require further investigation.
More than $30 \%$ of cancer patients will develop chronic pain associated with tumor growth and/or treatment, the majority of whom do not received adequate treatment (20). Opioids are the main pharmacological therapy for inhibiting cancer pain (21); however, adverse effects including nausea, constipation, urinary retention, dizziness and dysphoria are observed $(22,23)$. Porcellio laevis Latreille, the major component of JYQP, has been applied to ease liver cancer pain in 
clinical trials (12). In the present study, a significant analgesic effect of JYQP on rats with bone cancer pain was noted, indicated by an extension of the reaction time in a mechanical allodynia test and a reduction of the difference of bipedal pressure in a foot-supporting force test. Although previous data suggest that JYQP-mediated analgesic activity is associated with peripheral mediation (15), a further study will be performed to investigate the underlying mechanisms in detail.

As one of the most potent types of analgesics, opioids display a rapid onset of action and therapeutic effect on pain. Nevertheless, physical dependence has been noted following long-term drug administration (24). Physical dependence is a chronically relapsing disorder, which is characterized by a compulsion to seek and take the drug, loss of control in limiting intake, and the emergence of a negative emotional state, for example dysphoria, anxiety or irritability when the drug is not available (25). Addiction results in significant changes in mood and affective behavior, while withdrawal syndrome consists of severe somatic alterations (26). Morphine, a clear example, has significant inhibitory effects on numerous types of intense pain; however, the use of morphine is strictly controlled (27). In the present study, morphine caused a significant bodyweight loss after 30 days of administration in rats, and extremely high withdrawal behaviors were observed in morphine-dependent rats compared with non-treated animals. In JYQP-treated rats, no withdrawal symptoms were noted, indicating that JYQP is a reliable and safe agent for the alleviation of pain without addiction.

In conclusion, JYQP, a formulation composed of Porcellio laevis Latreille, Corydalis Rhizoma and Radix Cynanchi Paniculati, exhibited a significant analgesic effect in a rat model of bone cancer pain, as indicated by the positive results obtained in the foot-supporting force test and mechanical allodynia analysis. The chronic toxicity test and physical dependence assessment indicated that JYQP is a safe analgesic agent with no physical dependence following long-term administration. The JYQP formulation is patent-protected in China, and the data from the present study provide experimental support for the forthcoming clinical research.

\section{Acknowledgements}

This study was supported by the twelfth Five-Year National Key Technology R\&D Program of China(Grant no.2012BAL29B00), the Pharmaceutical Industry Development Special Fund of Jilin province, China (Grant no. 20130727013YY) and by funding from the Administration of Traditional Medicine of Jilin province, China (Grant no. 2011-zd18).

\section{References}

1. Bali KK, Selvaraj D, Satagopam VP, Lu J, Schneider R and Kuner R: Genome-wide identification and functional analyses of microRNA signatures associated with cancer pain. Embo Mol Med 5: 1740-1758, 2013.

2. Coleman RE: Clinical features of metastatic bone disease and risk of skeletal morbidity. Clin Cancer Res 12: 6243s-6249s, 2006.

3. Suh SY, Choi YS, Oh SC, et al: Caffeine as an adjuvant therapy to opioids in cancer pain: A randomized, double-blind, placebo-controlled trial. J Pain Symptom Manag 46: 474-482, 2013.
4. Mantyh PW: A mechanism-based understanding of bone cancer pain. Novartis Found Symp 261: 194-219, discussion 256-161, 2004.

5. Ali S, Mohs A, Thomas M, et al: The dual function cytokine IL-33 interacts with the transcription factor NF-kappaB to dampen NF-kappaB-stimulated gene transcription. J Immunol 187: 1609-1616, 2011.

6. Lai JN, Tang JL and Wang JD: Observational studies on evaluating the safety and adverse effects of traditional chinese medicine. Evid Based Complement Alternat Med 2013: 697893, 2013.

7. Yuan HW, Ma LX, Qi DD, Zhang P, Li CH and Zhu J: The historical development of deqi concept from classics of traditional chinese medicine to modern research: Exploitation of the connotation of deqi in Chinese medicine. Evid Based Complement Alternat Med 2013: 6393022013.

8. Li JC, Shen XF, Meng XL, Zhang Y and Lai XR: Analgesic effect and mechanism of the three TCM-herbal drug-combination Tou Feng Yu pill on treatment of migraine. Phytomedicine 18: 788-794, 2011.

9. Wang J, Zhang R, Dong C, et al: Topical treatment with Tong-Luo-San-Jie gel alleviates bone cancer pain in rats. J Ethnopharmacol 143: 905-913, 2012.

10. Gozum S, Tezel A and Koc M: Complementary alternative treatments used by patients with cancer in eastern Turkey. Cancer Nurs 26: 230-236, 2003.

11. Teng LR, Wang ZZ, Tian Y, et al: The preparation method of Armadillidium formula extract and the application of which in analgesic drugs. CN Patent 201110147689.7. Filed June 2, 2011; issued December 19, 2012.

12. Liang CY and Sun Z: The analgesic effect of Porcellio preparation on moderate to severe cancer pain. Shandong Zhongyi Zazhi 13: 159-160, 1994 (In Chinese).

13. Zhang XH, Lu TL and Mao CQ: Analgesic and anti-inflammatory effects of different kinds of Corydalis yanhusuo. Lishizhen Medicine and Materia Medica Research 20: 449-450, 2009 (In Chinese).

14. Xu QS, Zhang HY, Li YJ and Li D: Effect of the extract of Cynanchum paniculatum on anti-inflammation and analgesic in mice. Lishizhen Medicine and Materia Medica Research 18: 1407-1408, 2007 (In Chinese).

15. Tian Y, Teng LR, Song JJ, et al: Studies on the analgesic activities of Jia-Yuan-Qing pill and its safety evaluation in mice. Protoplasma 251: 1245-1253, 2014.

16. lbarrola DA, Hellión-lbarrola MC, Montalbetti Y, et al: Isolation of hypotensive compounds from Solanum sisymbriifolium Lam. J Ethnopharmacol 70: 301-307, 2000.

17. Chinese Pharmacopoeia Commision (eds): Pharmacopoeia Of The People's Republic Of China. Chinese Medical Science and Technology Press, Beijing, China, 2010.

18. Gao L, Liu G, Kang J, et al: Paclitaxel nanosuspensions coated with P-gp inhibitory surfactants: I. Acute toxicity and pharmacokinetics studies. Colloids Surf B Biointerfaces 111: 277-281, 2013.

19. El Hilaly J, Israili ZH and Lyoussi B: Acute and chronic toxicological studies of Ajuga iva in experimental animals. J Ethnopharmacol 91: 43-50, 2004.

20. Wilson KG, Chochinov HM, McPherson CJ, et al: Suffering with advanced cancer. J Clin Oncol 25: 1691-1697, 2007.

21. Krishna LK, Poulose JV, Tan BS and Goh C: Opioid use amongst cancer patients at the end of life. Ann Acad Med Singap 39: 790-797, 2010

22. Bowdle TA: Adverse effects of opioid agonists and agonist-antagonists in anaesthesia. Drug Saf 19: 173-189, 1998.

23. Stiefel F, Die Trill M, Berney A, Olarte JM and Razavi A: Depression in palliative care: a pragmatic report from the Expert Working Group of the European Association for Palliative Care. Support Care Cancer 9: 477-488, 2001

24. Lewis J, Mansour A, Khachaturian H, Watson SJ and Akil H: Opioids and pain regulation. Pain Headache 9: 129-159, 1987.

25. Koob GF: Neurobiological substrates for the dark side of compulsivity in addiction. Neuropharmacology 561: 18-31, 2009.

26. Crews FT and Boettiger CA: Impulsivity, frontal lobes and risk for addiction. Pharmacol Biochem Behav 93: 237-247, 2009.

27. Williams JT, Ingram SL, Henderson G, et al: Regulation of $\mu$-opioid receptors: desensitization, phosphorylation, internalization, and tolerance. Pharmacol Rev 65: 223-254, 2013. 